\title{
A novel biomarker protein panel for lung cancer, a promising first step
}

\author{
Camilo Molina-Romero, Edgar Vergara, Oscar Arrieta
}

Thoracic Oncology Unit and Experimental Oncology Laboratory, Instituto Nacional de Cancerología de México (INCan), Tlalpan, México City, Mexico

Correspondence to: Dr. Oscar Arrieta. Thoracic Oncology Unit, Instituto Nacional de Cancerología de México (INCan), San Fernando 22 Sección XVI, Tlalpan, Mexico City 14080, Mexico. Email: ogar@unam.mx.

Provenance: This is an invited Editorial commissioned by Section Editor Jun Zhou, MD (Department of Nuclear Medicine, Zhongshan Hospital, Fudan University, Shanghai, China).

Comment on: Guida F, Sun N, Bantis LE, et al. Assessment of Lung Cancer Risk on the Basis of a Biomarker Panel of Circulating Proteins. JAMA Oncol 2018;4:e182078.

Submitted Nov 05, 2018. Accepted for publication Nov 14, 2018.

doi: $10.21037 /$ tlcr.2018.12.09

View this article at: http://dx.doi.org/10.21037/tlcr.2018.12.09

Lung cancer (LC) is the most lethal cancer worldwide in both genders, tobacco consumption is causally associated with lung cancer, around $85 \%$ of all patients worldwide have tobacco consumption history $(1,2)$. The 5 -year survival of patients diagnosed at early stage and localized lung cancer is about $50 \%$, which is considerably better compared to later stages (3). However, around $61 \%$ of patients are diagnosed in advanced stages (III and IV), when the therapeutic options are limited and the prognosis is usually poor, with a 5 -year survival of only $5-15 \%(4,5)$. Even though important breakthroughs, progress in biomedicine, new diagnostic tools and the development of targeted scheme treatments, the main challenge with LC is to improve early detection of the patients, because according to different projections, the number of LC cases is expected to raise in the next years $(4,6)$.

The annual mortality rate for lung is higher than the mortality rates from other neoplasms, such as prostate, breast, and colon cancer, it should be noted that these neoplasms already have validated tools for early detection and diagnosis in early stages (7). A risk biomarker is defined as the one that can distinguish individuals at risk but who still do not have a measurable disease. A detection biomarker for early stages of the disease ideally allows to discriminate between groups of individuals at risk of developing the disease, additionally, must be able to distinguish the populations of individuals at risk before or after the disease is measurable (8). Nevertheless, any of these kind of biomarkers have been validated for LC.

Since the last half of the last century, several studies of screening with chest $\mathrm{X}$-rays and sputum cytology were evaluated, the result was the increasing number of lung cancer diagnosed without any improve of lung cancer specific mortality (8-10). High-risk profiles have been described for heavy smokers, who are defined as subjects with asbestos exposure, 30 or more pack-years of smoking history, or being older than 50 years, however, no models that use any biomolecular markers have been assessed and incorporated to clinical guidelines. Subjects at risk to develop LC, could be asymptomatic for years before being diagnosed. Therefore, the development of efficacious LC risk assessment models for use in this critical period capable to recognize high risk subjects and increase the rate of opportune detection and consequently initiate early treatment is a critical need (11).

The Early Lung Cancer Action Program started in the 1990's utilizing chest computed tomography (CT) imaging, it was a large lung cancer screening trial with success, which showed that the use of low-dose computed tomography (LDCT) to screen current or former heavy smokers can reduce lung cancer mortality $(12,13)$ and prompted the design of the National Lung Cancer Screening Trial (NLST), which results reported a $20 \%$ reduction in LC mortality, in high risk patients for LC after a median follow-up of 6.5-year, compared with conventional image 
screening (chest X-ray). Nowadays, the US preventive services task force (USPSTF) praises LDCT screening for LC among subjects with age between 55 to 80 years, with tobacco history (30 pack-years for at least 15 years), nevertheless, LDCT screening detects a considerable number of nonspecific nodules, in addition to the fact that about half of LC cases occur in subjects who are do not meet the criteria for screening (13-15).

The recent avenues in molecular biology strategies and the integration of analytical platforms, including "omics" approaches, have recognized several potential biomarkers in diverse biological samples (urine, saliva, sputum, blood, exhaled breath condensate, bronchial specimens), but none have yet received the approval to be included in the regular test panel for LC (8).

Last July, the Integrative Analysis of Lung Cancer Etiology and Risk (INTEGRAL) Consortium of Early Detection of Lung Cancer published the results of a LC risk prediction model based on a panel of selected circulating proteins combined with the traditional smoking history-based risk model. In the study, using one cohort, a blood based biomarker score was developed and compared against the traditional smoking history-based risk model alone, subsequently, it was externally validated using prediagnosis samples from two other independent cohorts (16). The results of this study revealed an overall specificity of 0.83 , grounded on the US prevention Services Task Force screening criteria, the sensitivity of the integrated risk prediction (biomarker) model was 0.63 , compared with 0.43 for the smoking model. On the other hand, furthermore, the integrated risk prediction model yield a specificity of 0.95 compared with 0.86 for the smoking model alone (1). The results of the study mark a potential improvement of the LDCT screening for the risk assessment of lung cancer, and represent a big step forward in the development and validation of a panel of biomarkers.

Four proteins were analyzed on these studies: cytokeratin-19 fragment (CYFRA 21-1), carcinoembryonic antigen (CEA), cancer antigen 125 (CA125), and the precursor form of surfactant protein B (Pro-SFTPB). CA125 is a glycoprotein produced in fetal tissue, it has been described that elevated serum CA125 levels are found in patients with seminal vesicle carcinoma, in ovarian cancer CA125 is utilized as a tumor marker for screening and management, furthermore, in LC, it has been reported the association of CA125 with bad prognosis (17). CEA is an oncofetal protein, member of the immunoglobulin family that is usually over-expressed in a number of neoplasms, including LC. Elevated serum CEA levels have been associated with advanced stages of NSCLC, brain metastases and poor prognosis (18). CYFRA 21 1 , a polypeptide that recognizes soluble cytokeratin, has been reported as the most sensitive biomarker to subtyping NSCLC and differentiating LC from non-maligns conditions (19). Pro-SFTPB is a circulating protein synthesized by nonciliated bronchiolar cells and type 2 alveolar pneumocytes. Pro-SFTPB is over-expressed in LC cells, particularly in adenocarcinomas; it has been reported that pro-SFTPB is a potential independent predictor of LC (20).

The main challenge before protein biomarkers panels can be used routinely in clinical practice is the need to validate their clinical utility. Although the study performed by INTEGRAL consortium provides good results with the first validation in different cohorts, the avenues to generate robust evidence to show clinical utility are still long and complicated. A prospective clinical trial, where the primary objective is the validation and calibration of the integrated risk prediction model of the biomarker panel, should be the ideal strategy, but conduct such trials is expensive and time-consuming (21). Other options are conduct what have been called prospective retrospective clinical studies, using patient specimens that have been collected and archived from previously conducted clinical trials that have addressed the potential use of the tumor biomarker test $(22,23)$, or, as the authors propose on this paper, the use of a larger prediagnostic sample size (16). Additionally, the biomarker panel path to the clinic use, is likely to be longer than the one for a single molecule, due to exacerbation of the typical difficulties related with biomarker development by merging "omics" methodologies (24). Proteomic technologies used in biomarker innovation are usually not transferable to clinical laboratories given to their high complexity, their low throughput and their analytical performance features (21), consequently, transferring the use of proteomic biomarkers from the investigative analysis phase to the clinical phase, require steady measuring platforms and prove to be economically viable $(21,24)$.

Having pointed out the difficulties that the panel still faces in its development, is important to highlight how biofluids-based markers analysis are a rapidly expanding area of biomedicine in translational cancer research, as it could be valuable in a wide range of applications, such as opportune diagnosis, prognosis data, stratification and follow-up of patients at real time, therapeutic targets, and resistance mechanism (25). Analysis of tissue samples are 
critical to identify a biological link among a biomarker and cancer risk, but standard tumor biopsies are not easily to acquire, they put the patient at risk, and might not accurately reflect the molecular alterations of tumors due to either suboptimal tissue acquisition or tumor heterogeneity, furthermore, biomarkers that involve biopsy are not pragmatic for assessing cancer risk or for the follow-up of the clinical response (24). Moreover, detection strategies based on the analysis of biofluids represent an appealing strategy for screening owed to offering a non-invasive attainment that ends up allowing a large number of samples available for analysis without any substantial risk for the patients, this would impact significantly the economic cost of the disease and its impact on health systems.

As conclusion, the results presented by the INTEGRAL consortium are encouraging, and it is necessary to validate this protein panel in order to stablish it as a stable, reproducible and non-invasive measured biomarkers panel.

\section{Acknowledgements}

None.

\section{Footnote}

Conflicts of Interest: Oscar Arrieta has received honoraria as advisor, participated in speakers' bureau and given expert opinions to Pfizer, AstraZeneca, Boehringer-Ingelheim, Roche, Lilly, and Bristol-Myers Squibb. Other authors have no conflicts of interest to declare.

\section{References}

1. Siegel RL, Miller KD, Jemal A. Cancer statistics, 2018. CA Cancer J Clin 2018;68:7-30.

2. Arrieta O, Quintana-Carrillo RH, Ahumada-Curiel G, et al. Medical care costs incurred by patients with smokingrelated non-small cell lung cancer treated at the National Cancer Institute of Mexico. Tob Induc Dis 2015;12:25.

3. Arrieta O, Pineda B, Muñiz-Hernández S, et al. Molecular detection and prognostic value of epithelial markers mRNA expression in peripheral blood of advanced non-small cell lung cancer patients. Cancer Biomark 2014;14:215-23.

4. Islami F, Torre LA, Jemal A. Global trends of lung cancer mortality and smoking prevalence. Transl Lung Cancer Res 2015;4:327-38.

5. Arrieta O, Guzmán-de Alba E, Alba-López LF, et al.
National consensus of diagnosis and treatment of nonsmall cell lung cancer. Rev Invest Clin 2013;65:S5-84.

6. Allemani C, Matsuda T, Di Carlo V, et al. Global surveillance of trends in cancer survival 2000-14 (CONCORD-3): analysis of individual records for 37513025 patients diagnosed with one of 18 cancers from 322 population-based registries in 71 countries. Lancet 2018;391:1023-75.

7. Brenner DE, Normolle DP. Biomarkers for cancer risk, early detection, and prognosis: the validation conundrum. Cancer Epidemiol Biomarkers Prev 2007;16:1918-20.

8. Hassanein M, Callison JC, Callaway-Lane C, et al. The state of molecular biomarkers for the early detection of lung cancer. Cancer Prev Res (Phila) 2012;5:992-1006.

9. Fontana RS, Sanderson DR, Taylor WF, et al. Early lung cancer detection: results of the initial (prevalence) radiologic and cytologic screening in the Mayo Clinic study. Am Rev Respir Dis 1984;130:561-5.

10. Stitik FP, Tockman MS. Radiographic screening in the early detection of lung cancer. Radiol Clin North Am 1978;16:347-66.

11. Gomperts BN, Spira A, Elashoff DE, et al. Lung cancer biomarkers: FISHing in the sputum for risk assessment and early detection. Cancer Prev Res (Phila) 2010;3:420-3.

12. Henschke CI, McCauley DI, Yankelevitz DF, et al. Early Lung Cancer Action Project: overall design and findings from baseline screening. Lancet 1999;354:99-105.

13. Aberle DR, Berg CD, Black WC, et al. The National Lung Screening Trial: overview and study design. Radiology 2011;258:243-53.

14. Muller DC, Johansson M, Brennan P. Lung Cancer Risk Prediction Model Incorporating Lung Function: Development and Validation in the UK Biobank Prospective Cohort Study. J Clin Oncol 2017;35:861-9.

15. Humphrey LL, Deffebach M, Pappas M, et al. Screening for lung cancer with low-dose computed tomography: a systematic review to update the US Preventive services task force recommendation. Ann Intern Med 2013;159:411-20.

16. Guida F, Sun N, Bantis LE, et al. Assessment of Lung Cancer Risk on the Basis of a Biomarker Panel of Circulating Proteins. JAMA Oncol 2018;4:e182078.

17. Isaksson S, Jönsson P, Monsef N, et al. CA 19-9 and CA 125 as potential predictors of disease recurrence in resectable lung adenocarcinoma. PLoS One 2017;12:e0186284.

18. Arrieta O, Saavedra-Perez D, Kuri R, et al. Brain metastasis development and poor survival associated with carcinoembryonic antigen (CEA) level in advanced non- 
small cell lung cancer: a prospective analysis. BMC Cancer 2009;9:119.

19. Korkmaz ET, Koksal D, Aksu F, et al. Triple test with tumor markers CYFRA 21.1, HE4, and ProGRP might contribute to diagnosis and subtyping of lung cancer. Clin Biochem 2018;58:15-9.

20. Sin DD, Tammemagi CM, Lam S, et al. Pro-surfactant protein $\mathrm{B}$ as a biomarker for lung cancer prediction. J Clin Oncol 2013;31:4536-43.

21. Füzéry AK, Levin J, Chan MM, et al. Translation of proteomic biomarkers into FDA approved cancer diagnostics: issues and challenges. Clin Proteomics 2013;10:13.

Cite this article as: Molina-Romero C, Vergara E, Arrieta O. A novel biomarker protein panel for lung cancer, a promising first step. Transl Lung Cancer Res 2018;7(Suppl 4):S304-S307. doi: 10.21037/tlcr.2018.12.09
22. Evaluation of Genomic Applications in Practice and Prevention (EGAPP) Working Group. The EGAPP initiative: lessons learned. Genet Med 2014;16:217-24.

23. Teutsch SM, Bradley LA, Palomaki GE, et al. The Evaluation of Genomic Applications in Practice and Prevention (EGAPP) Initiative: methods of the EGAPP Working Group. Genet Med 2009;11:3-14.

24. Vargas AJ, Harris CC. Biomarker development in the precision medicine era: lung cancer as a case study. Nat Rev Cancer 2016;16:525-37.

25. Rolfo C, Castiglia M, Hong D, et al. Liquid biopsies in lung cancer: the new ambrosia of researchers. Biochim Biophys Acta 2014;1846:539-46. 